\title{
LA CAMPAÑA DE LAURA CHINCHILLA Y LAS MUJERES ¿OPORTUNISMO O COMPROMISO CON UN NUEVO PACTO SEXUAL?'
}

\author{
LAURA CHINCHILLA CAMPAIGN AND WOMEN \\ OPPORTUNITIES OR UNDERTAKING A NEW COVENANT WITH SEX?
}

\section{María Flórez-Estrada*}

Si uno analiza, soy una mujer con una trayectoria similar a la de la mayor parte de mujeres costarricenses. Vengo de una familia de clase trabajadora, fui a una escuela pública, compartí con gente en el vecindario. He salido adelante con el apoyo de mi familia y, en lo fundamental, por un gran deseo de superación, como ocurre con la mayor parte de las mujeres.

Candidata presidencial Laura Chinchilla, 21 de enero de $2010^{2}$.

\section{RESUMEN}

En este ensayo analizo las articulaciones que ocurrieron entre la oferta electoral de la candidata Laura Chinchilla y la nueva realidad demográfica, social y sexual de Costa Rica, que ayudan a comprender por qué logró convocar el voto principalmente de las mujeres $y$ de las personas adultas mayores.

Además, planteo en qué consisten los nuevos desafíos de la sociedad costarricense en términos de un necesario nuevo pacto social y sexual, $y$ la perspectiva de su abordaje, o no, por el Gobierno de la primera Presidenta.

PALABRAS CLAVE: COSTA RICA * ECONOMÍA POLÍTICA * PARTICIPACIÓN DE LA MUJER * DIVISIÓN SEXUAL DEL TRABAJO * PARTICIPACIÓN POLÍTICA * TRANSICIÓN DEMOGRÁFICA

Agradezco a Ciska Raventós e Isabel Gamboa el haber leído una primera versión de este trabajo, que fue mejorado gracias a muchas de sus sugerencias. Y, por supuesto, las eximo de cualquier compromiso indeseado con el resultado final.

\footnotetext{
* $\quad$ Docente e investigadora del CIICLA. maria.florez-estrada@ucr.ac.cr

2 Vizcaíno, I. "Lo que viene es mi gobierno, no el de Óscar Arias”. 2010.
} 


\section{ABSTRACT}

In this paper, I analyze the connections that occurred between the electoral offer of candidate Laura Chinchilla and the new demographic, social and sexual realities of Costa Rica, which help to understand why mainly women and older people voted for her. In addition, I explain the new challenges the Costa Rican society faces in terms of a necessary new social and sexual contract, and the prospect of its approach, or not, by the Government of the first woman President.

KEY WORDS: COSTA RICA * POLITICAL ECONOMY * WOMEN`S PARTICIPATION * SEXUAL DIVISION OF LABOR * POLITICAL PARTICIPATION * DEMOGRAPHIC TRANSITION

El triunfo de Laura Chinchilla en las elecciones de febrero de 2010, por una mayoría indiscutible de los votos, no fue producto de la coyuntura ni únicamente del importante apoyo financiero que impulsó la campaña de la candidata del Partido Liberación Nacional (PLN).

Como he planteado en una investigación anterior ${ }^{3}$, los importantes cambios culturales, sociales y económicos que encaran la sociedad costarricense y la mayoría de los países del mundo, están afectando de manera importante las bases materiales y subjetivas que facilitaron la construcción de certezas personales y sociales en la segunda mitad del siglo Xx.

Pero, contrariamente a lo que se ha convertido en sentido común, estos cambios no obedecen única ni principalmente a la aplicación de medidas económicas neo-liberales o a la globalización. También tienen en su centro la gran revolución que las mujeres protagonizan en cuanto a sus lugares en el mundo, transformación que se expresa en decisiones culturales que impactan el mercado de trabajo, la demografía, las estructuras de los hogares, las relaciones entre hombres $y$ mujeres, pero sobre todo, plantea retos inusitados al sistema capitalista tal y como lo hemos conocido.

Mientras que la política tradicional en Costa Rica —incluida la de las izquierdas-, se ha seguido desarrollando en función de envejecidos ejes temáticos, en este artículo propongo

3 De "ama de casa" a "mulier economicus". Sexo, género, subjetividad y economía en Costa Rica contemporánea. Editorial de la Universidad de Costa Rica, 2010. que la candidatura de Laura Chinchilla —esto es, el que se tratase de una mujer, que además tuviera las características proyectadas durante la campaña-, y su oferta electoral de establecer una red universal de cuido para infantes $y$ personas adultas mayores, logró establecer vasos comunicantes, pero no únicamente con estos grupos del electorado.

\section{DECISIONES DE LAS MUJERES SOBRE SUS CUERPOS}

Una de las manifestaciones más importantes de la transformación protagonizada por las mujeres se expresa en la demografía, concretamente en la caída de la tasa de fecundidad: las jóvenes ya no tienen hijas e hijos entre los 20 y 35 años en la misma medida que en el pasado (históricamente, el grueso de los nacimientos provino de las de 20 a 29 años). Más aún, solo el tiempo dirá en qué medida esto corresponde a una decisión de postergar la maternidad o a un definitivo rechazo de esta.

Costa Rica es uno de los tres países de la región - los otros son Cuba y Chile-que se encuentran en una etapa de transición demográfica avanzada. La tasa de fecundidad en nuestro país ha llegado al nivel de reemplazo $(2,1$ hijos por mujer), pero hay factores que podrían acelerar su previsible descenso (CEPAL, 2008: 13$)^{4}$.

4 Las proyecciones realizadas con los datos de 2008 establecen la hipótesis de que la tasa global de fecundidad (TGF) observada en el 2007 de 1,96 hijos por mujer, continuará disminuyendo hasta alcanzar 1,70 en el 2015 y que luego, se incrementará hasta llegar a un valor de equilibrio de 1,90 a 
Lo anterior es viabilizado por un mayor acceso a los métodos de control de la natalidad no solo para las mujeres adultas, sino también para la población adolescente; por la permanencia más prolongada de las mujeres en el sistema educativo y por el aumento de la participación de las mujeres en el trabajo remunerado (CEPAL, 2008: 23-26).

Según la Asociación Demográfica Costarricense (ADC), el $80 \%$ de las mujeres que respondió una encuesta realizada en el 2007, utilizaba métodos anticonceptivos; el 51,6\% de las fértiles, no deseaba tener más hijas o hijos; el $77,9 \%$ de las fértiles que deseaba tener una hija o un hijo más, esperaba tenerlo hasta dentro de un año o más y el número promedio deseado de hijas o hijos era de 2,7\% (ADC, 2008: 11).

Ese año, de las 11632 mujeres que fueron atendidas por abortos, 8086 (70\%) lo fueron por las consecuencias de abortos inducidos ilegalmente (ADC, 2008: 56). Pero, la ADC calculó que, por cada mujer que aborta y busca atención médica, hay tres que no lo hacen, con lo cual los abortos ilegales ascenderían a 27000 por año (ADC, 2008: 52) 5 .

En cuanto al perfil de las mujeres que buscaron servicios para producirse abortos 0 para atender las consecuencias de habérselos producido, personal de salud entrevistado lo describió como "una mujer muy joven $(85,2 \%$ menores de 25 años); con educación secundaria o más (66,3\%); de zonas urbanas ( $80 \%)$; solteras $(82,5 \%)$ y sin hijos o con pocos hijos $(62,5 \%$ y 28,8\%), respectivamente" (ADC, 2008: 20).

La reducción en la tasa de fecundidad es una de las expresiones de cambios profundos ocurridos en la posición social de las mujeres en la sociedad y en sus subjetividades, lo cual a su vez está produciendo transformaciones en

partir del 2030 (CCP-INEC, 2008:14). Se calcula que el tamaño máximo de la población costarricense será de unos 6243000 personas, en el año 2055 , antes de comenzar a decrecer y estabilizarse (CEPAL, 2008: 23-26).

En el país, el aborto inducido únicamente es legal cuando está en peligro la vida y la salud de la mujer (Artículo 121). Código Penal.

En: <http://www.pgr.go.cr>. Consultado el 22 de octubre de 2010 la estructura de la familia tradicional, como se verá enseguida.

\section{DE “AMAS DE CASA" A MUJERES ECONÓMICAS ${ }^{6}$}

Otra de las transformaciones protagonizadas por las mujeres en los últimos treinta años tiene lugar en el mundo del trabajo, especialmente del trabajo pagado.

Mundialmente, la tasa neta de participación de las mujeres en el mercado laboral prácticamente se duplicó entre 1990 y 2005, y se estima que alcanzará el 50\% para el año 2030 (CEPAL, 2008: 86I) ${ }^{7}$.

En Costa Rica, a lo largo de los últimos veinte años, esa tasa pasó de un 30,4\% en 1988, a un 42\% en 2009 (Montiel, 2000 e INEC, 2009).

Mientras que en 1976, el 36,3\% de todas las mujeres del país eran "amas de casa", en el 2008 , lo eran el 25,5\%. Y mientras que hace treinta $y$ dos años, solo el $17,2 \%$ de todas las mujeres del país declararon tener una ocupación remunerada, se identificó, en el 2008, el 31,8\% (INEC, 1976 y 2008).

Es clara, pues, la tendencia a la reducción del número de mujeres "amas de casa", mientras que aumentan las que declaran tener una ocupación pagada.

Por otro lado, lo mismo que en el resto de América Latina (Arriagada, 2007: 129 y CEPAL, 2008: 127-128), en Costa Rica la composición de las familias es cada vez más diversa: se produce una disminución de los hogares nucleares constituidos por padre, madre, hijas e hijos, y aumentan los unipersonales y uniparentales, asociados al incremento en el número de separaciones y divorcios (Vega et ál., 2001: 204).

Un cálculo más reciente encontró que, mientras en 1990, los hogares costarricenses constituidos por un hombre proveedor y una

6 Ver más en: Flórez-Estrada, M. De "ama de casa" a mulier economicus. Sexo, género, subjetividad y economía en Costa Rica contemporánea. San José, CR. Editorial de la Universidad de Costa Rica, 2010.

$7 \quad$ La tasa neta de participación es la relación de la fuerza de trabajo con respecto a la población total de 12 años y más. 
mujer "ama de casa" eran el 63\% del total de hogares, para el año 2008 habían bajado al 38\% $y$ los biparentales con dos personas proveedoras - un hombre y una mujer - habían crecido del $19 \%$ al 35\%, lo cual es consistente con el incremento de las mujeres que trabajan remuneradamente. También, los hogares monoparentales de jefatura femenina crecieron del 18\% al $27 \%$ (Román et ál., 2010).

Pero, es importante resaltar que este cambio del paradigma de la mujer "ama de casa" por el de la "mujer proveedora remunerada", no ha significado, para ellas, una condonación social de las tareas de la reproducción doméstica, sino un recargo de funciones.

Es decir, que el pacto masculino de la segunda posguerra mundial, por el cual, en sus diversas versiones, el Estado de Bienestar prometía garantizar a cada trabajador un ingreso que le permitiera reproducirse a sí mismo, a "su" mujer y a "su" prole, se ha roto. Hoy, un solo proveedor no es suficiente para sostener un hogar, sino que, en los hogares nucleares, tanto el hombre como la mujer deben compartir el rol de personas trabajadoras pagadas, o de proveedoras para obtener ingresos, sin que este cambio haya implicado que ambos, hombres $y$ mujeres, compartan igualmente el trabajo reproductivo en el ámbito doméstico ${ }^{8}$.

Este cambio en la posición social de las mujeres también tiene consecuencias directas y con efectos de largo plazo, en la economía.

\section{LAS MUJERES DESACOMODAN EL CAPITALISMO}

Ilustremos el punto con la más reciente crisis financiera internacional. Poco antes de su estallido, en la segunda mitad de 2008, la preocupación era que los factores estructurales que afectan a una de las economías más influyentes del mundo $-y$ al principal mercado de las exportaciones centroamericanas-, amenazaban con propiciar un aumento de la inflación (incremento sostenido de los precios),

$8 \quad$ Esto es verificable por medio de las encuestas de Uso del tiempo que se vienen aplicando en diferentes países, incluido Costa Rica y a la que me referiré más adelante. debido a que la capacidad productiva de Estados Unidos, principalmente su fuerza de trabajo disponible, estaba utilizada en su totalidad (pleno empleo) y esto no era suficiente para satisfacer la demanda.

En febrero de ese año, cuando compareció ante el Comité de Asuntos Bancarios del Senado de EEUU para dar su Informe Semianual de la Política Monetaria, Ben Bernanke, presidente de la Reserva Federal (el Banco Central de ese país), advirtió que la crisis en la capacidad productiva de la economía estadounidense se debía a dos factores estructurales: i) el incremento en la participación laboral de las mujeres habría alcanzado ya su tope y ii) la generación del llamado baby boom comenzaba a jubilarse sin que se esperara un aumento en las tasas de reposición demográfica, debido a decisiones culturales (Bernanke, 2007).

Con el fin de comprender los desafíos que el capitalismo encara debido a los cambios en la posición social de las mujeres, detengámonos un momento en esta dimensión real de una de las economías más importantes del planeta, para lo cual es necesario, primero, llamar la atención sobre los límites androcéntricos con los cuales se construyó la ciencia económica.

Tanto los economistas clásicos como el marxismo invisibilizaron, en mayor o menor medida, el hecho de que la primera forma de organización económica o modo de producción consistió en la institución de un orden sexual conformado por dos clases de seres humanos: los hombres $y$ las mujeres (con sus desiguales valores $y$ libertades socialmente determinadas), y que la primera división del trabajo es la división sexual del trabajo.

Este orden primario, sobre el cual se construyen otras formas de opresión y explotación: esclavismo, servidumbre, capitalismo, tiene todo que ver con las crisis cíclicas del capitalismo y se hace cada vez más evidente ante la creciente significancia de las dimensiones demográfica y cultural de la crisis actual, como el Secretario del Tesoro estadounidense no tuvo más remedio que reconocer.

Porque, ¿qué significa, por una parte, que el incremento en la participación laboral de las mujeres, en Estados Unidos, habría alcanza- 
do ya su tope, de modo que, parte importante de la "sobrepoblación relativa" - en este caso, las mujeres aptas para el trabajo asalariado, pero "ociosas" (como si las "amas de casa" no trabajaran y produjeran el valor fuerza de trabajo) - que Marx calificó como parte del "ejército industrial de reserva", se agotó; esta vez, tal parece que para largo plazo? ${ }^{9}$.

Además, ¿qué significa, por la otra, que la generación del llamado baby boom haya comenzado a jubilarse sin que se espere un incremento en las tasas de reposición demográfica, debido a decisiones culturales?

Significa que la actual crisis del capitalismo está reactualizando el papel central que juegan, en el sistema, el orden sexual y la división sexual del trabajo. Nada menos que el hecho de que la economía ya no puede seguirse analizando y comprendiendo sin atender al papel relevante que la clase de las mujeres tiene para el capitalismo.

Como el propio Bernanke reconoce implícitamente para el caso estadounidense, por una parte, el capitalismo no podría existir sin ese trabajo gratuito, hoy por hoy realizado mayoritariamente por mujeres, en la reproducción de la fuerza de trabajo que será intercambiada en el mercado. De allí que la participación de las mujeres en el mercado de trabajo pagado - para cumplir con la función de "ejército industrial de reserva" durante las épocas expansivas de la economía-, tiene un límite dictado por esa necesidad de contar con un cierto número de "amas de casa" reproductoras de la fuerza de trabajo, límite que no existe para los hombres, esto es, para el "trabajador libre" del capitalismo.

$9 \quad$ "Pero si una sobrepoblación obrera es el producto necesario de la acumulación o del desarrollo de la riqueza sobre una base capitalista, esta sobrepoblación se convierte, a su vez, en palanca de la acumulación capitalista, e incluso en condición de existencia del modo capitalista de producción. Constituye un ejército industrial de reserva a disposición del capital, que le pertenece a este tan absolutamente como si lo hubiera criado a sus expensas. Esa sobrepoblación crea, para las variables necesidades de valorización del capital, el material humano explotable y siempre disponible, independientemente de los límites del aumento real experimentado por la población" (Marx, 2010).
Pero sucede que, al incrementarse de manera creciente la participación de las mujeres en el mercado de trabajo y también, al ser ellas cada vez más educadas, capacitadas y autónomas, no solo en EEUU sino en el mundo entero, se ha producido un cambio cultural cuyas manifestaciones en las subjetividades de las mujeres son patentes: ya no producen la fuerza de trabajo necesaria para que continúe la acumulación de capital y el capitalismo pueda seguir funcionando como lo conocimos hasta ahora.

Lo que encontré, por ejemplo, en la investigación antes citada, para el caso de mujeres profesionales y técnicas del sector público costarricense -mujeres de ingresos medios a altos-, es que se produce una postergación y subordinación de la maternidad a otras metas existenciales - como educarse, trabajar $y$ ganar dinero, comprar una casa, viajar, divertirse y "vivir bien"-, o es rechazada del todo (Flórez-Estrada, 2010).

Las jóvenes no quieren ser "amas de casa" como lo fueron sus propias madres y en varios casos son precisamente sus madres quienes las alientan a no repetir su historia. Como muestran los datos presentados más arriba, esto, a su vez, está produciendo un cambio demográfico: la tasa neta de fecundidad está al nivel o incluso por debajo de la tasa de reposición.

Por lo tanto, mientras las niñas y los niños que nacieron en la generación del llamado baby boom han envejecido y salen de la fuerza laboral —se jubilan-, las decisiones culturales que están tomando principalmente las mujeres, no garantizan que esa fuerza laboral pueda ser repuesta con nuevas generaciones.

Igualmente importante, implícitamente el jefe de la FED (Federal Reserve / Reserva Federal de EEUU) reconoció que el capitalismo estadounidense actual necesitaría de más mujeres trabajando remuneradamente o, en todo caso, de una fuerza de trabajo más numerosa, para atender la demanda global en tiempos de actividad económica de pleno empleo ( $y$ ya sabemos de sus restricciones migratorias que, si bien no le permiten aumentar suficientemente el "ejército 
industrial de reserva", le sirven para abaratar el costo de esta fuerza de trabajo adicional).

Esto significa que el "pacto" sexual implícito en el modelo típico de "burgués y proletario" y de "hombres-proveedores con esposatrabajadora-doméstica-gratuita-productora de prole para intercambiar en el mercado", del capitalismo industrial, ya no da para más.

Se ha roto el pacto sexual y social sobre el que se construyó el capitalismo de la posguerra (en el caso de Costa Rica, realizado mediante la patriarcal alianza calderonista, católica y comunista).

Mientras que, bajo aquellos: Estado, patronos $y$ trabajadores garantizaban tripartitamente la provisión de garantías sociales o de ese "salario familiar" suficiente para la reproducción del trabajador (literalmente) y de su familia, a lo largo de sus vidas (la promesa del Estado del Bienestar), con la puesta en efecto del nuevo modelo neoliberal, en la década de los 80 del siglo Xx, el viejo pacto acentuó su fractura.

El ataque a los salarios mínimos fue el medio por el cual se abarató el costo de la fuerza de trabajo como parte de la decisión política de realizar cambios estructurales, en el sentido de retirar al Estado de la economía y de ampliar el espacio para la acumulación privada: se garantizó "a los asalariados" (literalmente) un ingreso mínimo que les permitiera cubrir "apenas una tercera parte de los bienes y servicios tomados en cuenta para calcular el Índice de Precios al Consumidor (Lizano, 1999: 77-78)"10.

Lo anterior tuvo un impacto en los ingresos de los hogares, que provocó el efecto de "trabajador añadido", esto es, que los hogares ya no pudieran subsistir con un solo proveedor y este trabajo remunerado "adicional" — según el paradigma androcéntrico- fue

10 El mismo autor y Presidente del Banco Central a lo largo del período de las reformas, explica que superada la crisis, progresivamente se aumentaron los productos incluidos en la canasta básica $y$ que podían ser adquiridos con el salario mínimo, hasta tomar como referencia al Índice de Precios al Consumidor (IPC). Pero, la "canasta básica alimentaria" elaborada a partir de las costumbres de consumo de 1988, recién fue actualizada en el año 2010 . y todavía es provisto principalmente por las mujeres.

En adelante, para una gran mayoría de hogares, la identidad masculina y la autoridad patriarcal ya no se podrían construir en torno a la función del "proveedor", ni las mujeres podrían vivir en el estado de abstracción de las "amas de casa", sino que, además de cumplir con el trabajo doméstico no remunerado, tendrían que salir del claustro privado a trabajar remuneradamente y convertirse en actoras económicas y políticas.

El paradigma clásico del homo economicus comenzaría a enfrentar los dilemas y desafíos de la mulier economicus, lo cual, en términos de la división sexual del trabajo nos coloca en un escenario más parecido — por supuesto, salvando las diferencias - al mundo del capitalismo preindustrial, en el cual la distinción hombre proveedor/ mujer "ama de casa" era más difusa" ${ }^{11}$.

Nos encontramos, pues, ante la necesidad de negociar un nuevo acuerdo, no solo en términos de "modelo de desarrollo", sino en cuanto al orden sexual tradicional. Pues es bastante dudoso que, tras la destrucción masiva de empleos por las crisis cíclicas del capitalismo, las mujeres que se han educado y capacitado y que quieren ser autónomas económicamente a través del trabajo remunerado, acepten volver al viejo rol de "amas de casa" y cerrar sus horizontes a lo que muestra la ventana de su hogar, incluso si esta se ubica en lo alto de una loma con incomparable vista, en un confortable barrio residencial de clase media ${ }^{12}$.

11 Sobre este proceso y la construcción del mito de la "mujer trabajadora" del capitalismo industrial puede verse el excelente trabajo de Scott (2000).

El derecho al trabajo remunerado de las mujeres suele ser ignorado especialmente en épocas de crisis, pues las empresas optan por despedirlas primero a ellas y a las personas jóvenes, la mitad de las cuales son mujeres, como si ese derecho fuera, sobre todo, una prerrogativa masculina. Con la última crisis, la tasa de desempleo femenino a nivel mundial aumentó de 6,0 por ciento en el 2007 a 7,0 por ciento en el 2009, mientras que la masculina creció de 5,5 a 6,3 por ciento (OIT, 2010). 


\section{LAURA CHINCHILLA: UNA MUJER EDUCADA}

Puede afirmarse que el triunfo electoral de Laura Chinchilla, en tanto primera mujer Presidenta de la República, coronó un proceso de 120 años de lucha de las mujeres costarricenses por la igualdad de derechos políticos, en el marco del feminismo liberal y cuyas iniciadoras históricas fueron las sufragistas. Creo que puede decirse esto a partir del hito alcanzado, pero sin que ello signifique que exista completa o, siquiera, cercana igualdad y equidad entre hombres $y$ mujeres en el terreno de la lucha política ${ }^{13}$.

No hay acceso, todavía, a datos del Tribunal Supremo de Elecciones sobre la votación general de 2010 desagregada por sexo. Sin embargo, una encuesta telefónica realizada por el Centro de Investigaciones y Estudios Políticos (CIEP) y la Escuela de Sociología, de la Universidad de Costa Rica, después de los comicios del 7 de febrero de 2010, encontró que la victoria de Chinchilla se debió, principalmente, a que las mujeres y las personas adultas mayores (de las que podemos suponer que al menos la mitad son mujeres), le dieron su respaldo ${ }^{14}$.

El 53,2\% de las mujeres electoras la escogió (frente a un 19,4\% que apoyó a Ottón Solís y a un $12,8 \%$ que sufragó por Otto Guevara). Además, la apoyaron personas de todas las edades, pero, a más edad, en mayor proporción votaron por ella, en el siguiente orden: $58,5 \%$ de más de 60 años; 53,7\% entre 41 y 60 años; 47,8\% entre 26 y 40 años; y 37,2\% entre 18 y 25 años. Este respaldo generalizado de las mujeres, pero

13 Aunque no es un tema que desarrollaré aquí, uno de los debates más interesantes dentro del gran debate electoral pasado es el que se dio entre feministas que no apoyaban a Laura Chinchilla debido a su filiación ideológica y quienes reivindicaron el papel del Partido Liberación Nacional en los avances de las luchas políticas feministas que hicieron posible que una mujer aspirara a la Presidencia del país. Sobre esto último, puede verse, por ejemplo, el artículo "Liberación Nacional y las mujeres", de Juan Rafael Espinoza, publicado en el diario La Nación, el 17 de diciembre de 2009.

14 La encuesta se realizó del 8 al 12 de febrero de 2010 con 1205 entrevistas válidas, un margen de error de 2,8\% y un nivel de confianza del $95 \%$. Según el INEC, en 2009 el 65,7\% de los hogares costarricenses tenía teléfono. sobre todo de las mayores, puede tener como uno de sus sustentos precisamente la memoria histórica y los avances de las luchas feministas por los derechos políticos en Costa Rica ${ }^{15}$.

Otro sustento de este voto tendría que ver con lo planteado líneas arriba, sobre el creciente protagonismo de las mujeres en la vida económica y política, lo cual es consistente con lo encontrado por Ciska Raventós et ál.:

El voto femenino ha crecido de tal forma que en muchos países hoy votan más las mujeres que los hombres ... En Costa Rica votan más las mujeres que los hombres desde 1994 y, al igual que los Estados Unidos, la tendencia a la abstención masculina es creciente (Raventós et ál., 2005: 27).

De hecho, según los datos del TSE, la tendencia al incremento del abstencionismo no solo se detuvo, sino que se redujo durante los pasados comicios, pues bajó 4,1 puntos (de $34,8 \%$ en los comicios de 2006 a 30,9\% en 2010) (Consultado en : <http://www.tse.go.cr/ elecciones.htm>), muy probablemente debido a la posibilidad de que una mujer alcanzara la Presidencia de la República (cuando los datos estén disponibles, será posible saber el papel que el voto femenino mismo jugó en el freno y reducción de la tendencia abstencionista).

Además, según la encuesta, la Presidenta Chinchilla recibió la mayoría de votos por las siguientes razones: su capacidad y personalidad $(29,0 \%)$, sus propuestas de campaña y la campaña desarrollada por ella $(18,3 \%)$ y por ser mujer (15,7\%) (CIEP-UCR, 2010). Es decir, que el hecho de que Laura Chinchilla fuera mujer tuvo un peso específico propio en la votación. En contraste, ninguna de las personas electoras que respaldó a sus rivales dijo haber votado por ellos expresamente porque fueran hombres. Lo anterior implica que el electorado no naturalizó la candidatura presidencial de

$15 \quad$ Evidencia empírica de esta memoria encontró, por ejemplo, una investigación de Gamboa Barboza (2009: 29). Las personas entrevistadas por ella, hombres y mujeres costarricenses nacidas en la primera mitad del siglo XX, tenían una memoria muy fresca $y$ de respaldo, sobre la conquista del derecho al voto por las mujeres. 
una mujer costarricense - cosa que sí ocurrió con las candidaturas masculinas-y que al menos una parte de las personas electoras se movilizó expresamente para viabilizar que una mujer lograra el máximo cargo político. Esto, independientemente de que tuviera o no expectativas de que, por el hecho de ser mujer, aportaría un cambio político de importancia.

Puede resumirse entonces, que el hecho de que Chinchilla fuese una mujer, que se proyectara con personalidad y capacidad, unido a sus propuestas de campaña y a la forma como desarrolló esta, fueron los factores que determinaron la preferencia de la mayoría que la escogió. Lo anterior, sin dejar de considerar el respaldo que recibió expresamente por su filiación liberacionista ${ }^{16}$.

Ya me he referido suficientemente a los datos que sustentan la relevancia $-y$ no la "casualidad" - del hecho que la ganadora de la lucha por la Presidencia fuese una mujer.

16 Como dije antes, el hecho de que una mujer del PLN fuese candidata presidencial con opción de poder generó una fuerte crítica de parte de feministas, quienes la acusaron de atribuir a su partido éxitos que no le corresponderían, de ocultar las luchas feministas, e inclusive de estar subordinada al expresidente Óscar Arias, debido a su vínculo político (Informatico, 2010). Paradójicamente, con este último argumento, igual que la campaña desarrollada por el Partido Acción Ciudadana (PAC) para proyectar a Chinchilla como una marioneta de Arias, le negaron a la candidata su propia capacidad de agencia, al peor estilo misógino. Lo cierto es que, ya en el ejercicio del poder, Chinchilla dio muestras de su autonomía relativa al interior del PLN. Por otro lado, tampoco la exdiputada $y$ pre-candidata presidencial del PAC, Epsy Campbell, reivindicó el feminismo como parte de sus compromisos mientras ejerció como diputada o disputó la candidatuta a Ottón Solís, pero esto no fue objeto de su descalificación pública por feministas. Otro argumento usado para desacreditar a Chinchilla fue afirmar que se proyectaba de manera masculinizada en la competencia electoral, razonamiento comprensible en las feministas de la diferencia, pero no, por ejemplo, en las feministas radicales, pues para estas últimas se estaría negando la realidad de que, en términos de género, existe una gran diversidad de mujeres. Lo cierto es que la diversidad misma del feminismo impide que este interprete de manera unívoca la elección de Chinchilla.
Ahora me referiré, brevemente, a otro de los factores que más incidió en sus simpatizantes: su capacidad $y$ personalidad.

Como Laura Chinchilla se percibe a sí misma en la cita que abre este artículo, ella se parece a esas miles de mujeres que, especialmente desde la segunda mitad del siglo xx, han escalado en su autonomía profesional y económica gracias a la educación.

Educarse más que los hombres es una de las estrategias que las mujeres despliegan para sortear los obstáculos que son puestos en su camino con el fin de discriminarlas en la competencia por obtener el reconocimiento económico y simbólico en el mercado laboral costarricense ${ }^{17}$.

Las mujeres costarricenses estudian más que los hombres en todas las etapas y modalidades de la educación formal. Además, el análisis de una serie de catorce años (1990-2004), de las EHPM (Encuesta de Hogares de Propósitos Múltiples), muestra una clara tendencia a que un mayor número de mujeres se gradúe de la universidad en relación con los hombres (una razón mujeres/hombres de 130,9 en 1990 y 159,3 en 2004) (Flórez-Estrada, 2009).

A la afirmación "obtener una educación universitaria es más importante para un hombre que para una mujer", de la Encuesta Nacional de Convivencia (ENCON-2008), realizada en Costa Rica por el Programa de las Naciones Unidas para el Desarrollo, el 67,9\% de las mujeres dijeron estar "en desacuerdo", frente al $66,7 \%$ de los hombres. Pero el $17,8 \%$ de las mujeres dijo estar "muy en desacuerdo", frente al $12,8 \%$ de los hombres.

A la afirmación "cuando el desempleo es alto se le debe dar prioridad a los hombres en los trabajos", el 59,2\% de las mujeres dijo estar "en desacuerdo", frente al 57,5\% de los hombres. Pero, el 19,1\% de los hombres dijo estar "de acuerdo", frente al 17,1\% de las mujeres.

El mayor rechazo de las mujeres a una discriminación en su contra $y$ en beneficio de

17 Ejemplos de esto pueden verse en los resultados de la investigación que realicé en dos empresas de inversión extranjera directa y una de capital nacional, establecidas en el Régimen de Zona Franca (Flórez-Estrada, 2007). 
los hombres, en cuanto a su acceso a la educación universitaria y al trabajo remunerado, pero también el hecho de que la mayoría de los hombres entrevistados se sumaran a este rechazo, indica el grado de legitimidad que estos derechos, como derechos también de las mujeres, han alcanzado en la sociedad costarricense.

Además, se trata, como se ve, de otro elemento que indica la ruptura del viejo "pacto" sexual y social, y que posibilita el proceso de transición que caracteriza a la sociedad costarricense contemporánea: la igualdad del derecho a la educación y la igualdad del derecho al trabajo remunerado, para las mujeres, que tiende a naturalizarse, a hacerse "sentido común" de hombres y mujeres, pero especialmente de las mujeres. Esto, a pesar de que todavía hay un significativo grupo de mujeres $y$ hombres que siguen pensando de la manera tradicional.

De quienes votaron por Laura Chinchilla, un $18,5 \%$ cursó la universidad, un porcentaje igual el colegio y un $25 \%$ la primaria, mientras que un $2 \%$ no tenía estudios (Luego veremos por qué la oferta de la red de cuido pudo atraer el voto de las mujeres tanto profesionales como de las de menor educación y pobres, con carga familiar).

Si se comparan estas cifras con las obtenidas por su rival más cercano en su vasta lejanía, hay que decirlo, podría suponerse que el sentido común prevaleció en la preferencia por Chinchilla (además de ser mayoritariamente hombres, el $30 \%$ de quienes apoyaron a Ottón Solís tienen estudios universitarios completos, el 17,6\% completó la secundaria y el $14,3 \%$ la primaria, mientras que un $0,4 \%$ carece de estudios).

Puede que el sentido común prevaleciera sobre el pensamiento crítico (suponiendo que cursar la universidad efectivamente garantice este último), pero, también sabemos que en los procesos electorales, lo último que debe subestimarse es precisamente, el sentido común, que en este caso estuvo fuertemente vinculado al avance de las mujeres, que son la mitad de la población. Fue, por tanto, un sentido común, en alto grado - aunque solo fuese intuitivamente, puesto que es sentido común-, feminista.
Pero, ¿cómo se articuló este sentido común feminista con el programa electoral de Chinchilla? ¿Cómo logró convocar también a los hombres y personas adultas mayores? ¿Se trató de un sentido común progresista o conservador?

\section{LA RED NACIONAL DE CUIDO COMO PROGRAMA ELECTORAL}

Como expliqué más arriba, la ruptura del "pacto" sexual y social de la post-guerra implicó que las mujeres también se volvieran proveedoras económicas de primer orden de sus hogares, pero no implicó que los hombres se volvieran reproductores $y$ cuidadores obligados de sus familias ${ }^{18}$.

Es decir, que mientras que las mujeres se han visto obligadas a aumentar el peso de su trabajo remunerado para llevar ingresos a sus hogares, socialmente no se las ha eximido del trabajo de la reproducción y el cuidado de la infancia, la fuerza laboral y de las personas adultas mayores y discapacitadas. En otras palabras, la carga global de trabajo (remunerado más no remunerado) ha crecido para ellas, no así para los hombres.

Las organizaciones de mujeres $y$ de feministas, de todo el mundo, saben esto desde hace mucho y por eso están tratando de institucionalizar la aplicación de encuestas de uso del tiempo por sexo, como parte del esfuerzo por hacer que se visibilice y valore el aporte económico y vital de las mujeres a la economía.

Como producto de este esfuerzo, en Costa Rica, el INEC por primera vez aplicó un Módulo de Uso del Tiempo con la EHPM de 2004, cuyos resultados son elocuentes.

Dependiendo de su ocupación principal, las mujeres tienen una carga global de trabajo (CGT) superior a la de los hombres en casi 14 horas semanales $(13,54$ horas semanales para las que trabajan en la agricultura y la pesca).

18 Me refiero a que se produjo un cambio en el paradigma, pues, históricamente, la gran mayoría de las mujeres han aportado ingresos a sus hogares, incluso cuando se las clasificaba como "amas de casa". 
Más aún, el hecho de que las mujeres alcancen un alto grado de educación, no les garantiza un alivio de esta carga, pues las mujeres profesionales, científicas e intelectuales registraron una CGT de 10,46 horas semanales más que los hombres de su mismo grupo (AGEM et ál., 2010).

Esta sobrecarga de trabajo para las mujeres constituye una de las limitaciones clave para que ellas puedan competir en igualdad de condiciones con los hombres en el mercado laboral, tiene consecuencias en su salud y las limita para avanzar en su propia formación ${ }^{19}$.

La necesidad de que la reproducción y el cuidado de las personas fuesen reconocidos como trabajos socialmente necesarios los cuales, por tanto, deben ser socialmente asumidos $y$ no cargados únicamente a las mujeres, es una reivindicación que gracias a sus luchas se logró integrar, en el año 2007, como el primer punto del Plan de Acción de la Política Nacional de Igualdad y Equidad de Género (PIEG) del INAMU $^{20}$.

Un estudio reciente encontró que entre 1990 y 2008 aumentó en más del doble el número de niñas y niños en los hogares biparentales "con doble proveedor", pues pasaron de 59582 a 161 200. Tales hogares concentran el 36\% de la población de niños y niñas. Otro 18\% de infantes viven en hogares monoparentales con jefatura femenina (80 361) y también se duplicaron desde 1990. Si bien el estudio encontró que los hogares biparentales con hombre proveedor y cónyuge ama de casa siguen concentrando la mayor cantidad de infantes (204 654), disminuyeron de manera

19 Las mujeres estudian más que los hombres hasta el grado de licenciatura y maestría, pero los hombres adquieren más doctorados, lo cual puede coincidir con los compromisos de la reproducción y cuidado de hijas e hijos (Flórez-Estrada, 2009).

"I. Cuido como responsabilidad social. Que en el 2017 toda mujer que requiera servicios de cuido de niñas y niños para desempeñarse en un trabajo remunerado, cuente con, al menos, una alternativa de cuido pública, privada o mixta de calidad, dando así pasos concretos hacia la responsabilidad social en el cuido y la valoración del trabajo doméstico" (INAMU, 2008). importante con respecto a 1990, pues pasaron del 72\% al 46\% (Román et ál., 2010: 14).

El equipo de campaña del PLN ha de haber hecho sus números sobre el voto potencial de las mujeres y de las personas adultas mayores, pues la candidata Laura Chinchilla tuvo el sentido de oportunidad de apropiarse de esta necesidad no atendida $y$ de darle un lugar destacado en su programa electoral, como su segundo objetivo, después del tema de la seguridad ciudadana.

Bajo el título "Bienestar y protección de los más vulnerables" propuso tres programas sociales, el primero de los cuales fue el:

Programa Nacional de Cuido, para niños, niñas y personas adultas mayores, que ofrezca a la niñez el cuido y la estimulación temprana que son cruciales para el éxito escolar más adelante en sus vidas, $y$ a los adultos, especialmente las mujeres jefas de hogar, la oportunidad de trabajar fuera del hogar y ganar los ingresos que necesitan para sacar adelante sus familias (Consultado en: $<$ http://www.archivoelectoral.org/ documentos/11>21.

Ni el PAC, ni la izquierda, ni ningún otro partido dieron a este tema la relevancia que tiene ${ }^{22}$.

21 Que se trató de un "sentido de la oportunidad" u "oportunismo electoral" - para decirlo en jerga no académica - de la campaña de Chinchilla, quedó demostrado una vez que comenzó a gobernar: aparte del enunciado, la Presidenta no había previsto ni cómo hacer esta Red ni cómo financiarla. Sobre esto puede leerse: "Gobierno dedicará $\$ 32000$ millones para redes de cuido" (Mata, A. 2010). En: <http://www.nacion.com>. Consultado el 5/10/10. Sin embargo, también el artículo "La familia y el cuidado de dependientes", firmado por Chinchilla y publicado en agosto de 2009 , muestra que su equipo de campaña manejaba los argumentos contextuales sobre las transformaciones ocurridas en el mundo del trabajo. Puede leerse en: <http://www.nacion.com>. Consultado el $20 / 10 / 10$.

22 De hecho, también antes que Chinchilla, la Asociación Nacional de Empleados Públicos y Privados (ANEP) incluyó una propuesta similar 
El efecto convocante y multiplicador de simpatías de esta oferta electoral puede deducirse en la medida que, además de dirigirse a atender las necesidades materiales de las mujeres que trabajan remuneradamente, de las "amas de casa" que no pueden emplearse porque no pueden pagar a otras personas para que asuman estos trabajos de reproducción y cuidados, de las propias niñas y niños (cuyo papel activo en la campaña pro-Chinchilla fue muy visibilizado) y de las personas adultas mayores y discapacitadas, "en esa oferta está implícito un compromiso con la reconstitución de la familia tradicional costarricense", la cual, como han mostrado los datos, pasa por profundas transformaciones ${ }^{23}$.

para encarar la crisis, pero como el cuarto punto de su plataforma " 10 medidas para enfrentar la crisis económica con inclusión social y productiva", de 2009, bajo el título "Corresponsabilidad social y trabajo decente mediante infraestructura social de los cuidados" (Consultado en <http:// www.anep.or.cr/leer.php/2914>)".

Traté de encontrar un referente teórico para esta clase de "articulación" entre un electorado y una oferta electoral, pero, o se ponía más el peso en la racionalidad de los intereses, como en Althousser (Jameson et ál., 1998 y Barciela, 2007) y en el lenguaje y el discurso Foucault (1985 y 1999); o en la "identidad", particularmente "colectiva", como en Polleta et ál. (2001). (Agradezco las sugerencias de Alexander Jiménez y Ciska Raventós en esto). Sin embargo, esta clase de articulación, que no necesariamente configura, ni siquiera en una coyuntura, una "identidad" social o colectiva y que tampoco es un proceso discursivo o políticamente consciente, se aproxima más al "magma de significaciones imaginarias sociales" de Castoriadis (1989), porque se nutre "intuitivamente" — no racionalmente- de un imaginario compartido, aunque no es vivido ni desde lugares sociales semejantes, sino, sobre todo, desde lugares sociales y subjetivos muy diversos e incluso divergentes. Esto estaría explicado, hoy, precisamente por la gran desarticulación tanto de la vida privada como de las "certezas" sociales y personales a las que hice referencia al comienzo. En todo caso, hay más proximidad entre el magma castoriadiano y un electorado que se articula puntualmente para cumplir la "función autor" — como articuladora del imaginario social en y para un momento electoral-, como el que, según Foucault (1999: 30-31), la modernidad demanda, porque reconoce que toda articulación y todo autor, están atrave-
En la medida que el efecto buscado por la Red de Cuido es - como afirman la PIEG y el Plan de Gobierno de Chinchilla- principalmente facilitar que las mujeres trabajadoras cumplan de una manera más eficiente, en las nuevas condiciones, con el tradicional papel de madres; es decir, de reproductoras y protectoras de la futura $y$ presente fuerza de trabajo, $y$ en la medida que no hay un cuestionamiento de la división sexual del trabajo, ni de la maternidad como destino social de las mujeres, sino una "promesa" de reasegurarla, esta propuesta del programa de Chinchilla, en su carácter conservador - de protección y rescate de los valores tradicionales costarricenses-, explicaría su poder de apelar no solo a las mujeres, sino también a muchos hombres.

De hecho, en el artículo de Chinchilla antes citado, publicado seis meses antes de las elecciones, el acento está puesto en reafirmar el papel de la tradicional familia costarricense y lleva implícita la promesa de rescatarla de los embates a que es sometida por la creciente incorporación de las mujeres al trabajo extradoméstico (este énfasis también se refleja en que la palabra "familia" o "familiares" es utilizada 14 veces, mientras que la palabra "mujeres", solo cuatro veces).

El artículo finaliza explicitando el fin último de la "red de cuido":

Si queremos que las familias costarricenses del siglo XXI se fortalezcan y formen personas sanas física $y$ emocionalmente, es preciso incorporar a otros actores sociales y económicos de nuestra sociedad en la vital tarea de cuidar a nuestros niños y niñas, a las personas enfermas o discapacitadas, $y$ a las que están en edad avanzada (<http://www.nacion.com>. Consultado el 17/08/10) ${ }^{24}$.

sados por esa creación social anónima. Podría, entonces, definirse, como una "articulación no identitaria de sentidos comunes", en coyunturas electorales.

24 Nótese, por lo demás, que este llamado a socializar el cuido hubiera sido tildado de "comunista" en el contexto de la Guerra Fría. Por otro lado, un estudio de la UCR reveló, recientemente, que el 
Una promesa de rescate que, sin duda, resultó convocante especialmente para los sectores de ingresos bajos $y$ medios ${ }^{25}$.

\section{EPÍLOGO: ¿IGUALDAD PARA TODAS Y TODOS?}

En este artículo he procurado mostrar los procesos sociales por los cuales fue posible que una mujer llegara a la Presidencia de la República, en Costa Rica, con el voto mayoritario de mujeres y de hombres.

Al mismo tiempo, contrariamente a la interpretación feminista que desvinculó el ascenso político de Laura Chinchilla de los importantes procesos sociales protagonizados históricamente por las mujeres costarricenses, he rescatado, para el feminismo, la importancia de este hecho, independientemente del partido del cual proviene Laura Chinchilla y de mi propia posición política e ideológica.

No creo que contribuya a las luchas feministas rebajar nuestros propios logros cuando no compartimos el programa político ni la ideología de la ganadora de los comicios ${ }^{26}$.

consumo de frijoles entre los ticos disminuyó un $35,1 \%$ desde 1965, "con lo cual estarían perdiendo la protección contra el cáncer y males cróni$\cos y$ cardiovasculares que provee esta leguminosa" (Rodríguez, 2010). La investigadora, Hannia Campos, dijo a la periodista que la baja en el consumo se debe a que "el estilo de vida cambió y las mujeres no tienen tiempo para cocinar los frijoles. Pero este cambio de vida va en detrimento de su salud" (Ídem). Con información como esta puede comprenderse lo apelante que ha de ser la oferta de recomponer la familia tradicional costarricense $y$ ojalá, devolver a las mujeres a la cocina.

Ciska Raventós me recordó que también el PAC recurrió, durante la campaña electoral del 2006 , a la figura de la madre como elemento central. El hecho de que casi ganara las elecciones en esa oportunidad solo ratifica cuán apelante resulta para amplios sectores la idea de un retorno a la familia paradigmática del Estado Social. Es decir, que desde la perspectiva del "contrato" sexual, no solo la derecha puede ser profundamente conservadora.

Por ejemplo, a pesar de que es un hecho probado que la candidatura de Laura Chinchilla pudo materializarse al interior del PLN en buena medida gracias al respaldo político que le diera Óscar
También he expuesto que los cambios que han tenido lugar en la posición social de las mujeres son complejos y paradójicos, pues significan avances en su autonomía, pero no necesariamente implican la superación de la división sexual del trabajo - antes bien, sí implican una sobrecarga de trabajo para las mujeres.

En este sentido, que el Estado financie una red de soporte para el cuidado de infantes y personas adultas mayores, implicará atender una necesidad apremiante para muchas mujeres, tanto profesionales de los sectores medios como, especialmente, de las más pobres y con menor nivel educativo, aunque, sin otras políticas públicas destinadas a transformar las ideas tradicionales sobre los hombres y las mujeres, también contribuirá a reafirmar la división sexual del trabajo.

Es decir, que revitalizar la vieja política de apoyo a la maternidad, del Estado de Bienestar, puede enmarcarse en procesos conservadores o progresistas en términos del derecho de las mujeres a no ser socialmente subordinadas ni objeto de discriminación en el mundo del trabajo o en otros órdenes de la vida social.

He mostrado evidencia de que el viejo "pacto" sexual y social de ese Estado, basado en el paradigma del "hombre proveedor" y la mujer "ama de casa", ha llegado a su fin y que el reto es sustituirlo con un nuevo acuerdo social y sexual que, esta vez, ha de tomar como protagonistas e interlocutoras a las propias mujeres, que ya no son subjetivamente las mismas del pasado.

Dada la posición expresamente conservadora asumida por la presidenta Chinchilla, desde su discurso inaugural en el cargo, existe más evidencia para afirmar que su Gobierno se enmarca en un proceso conservador, de reacción a los profundos cambios culturales que han ocurrido en las últimas décadas, que en un proceso progresista, de afirmación de los dere-

Arias, incluso frente a la oposición de su hermano Rodrigo - y al hecho de que Óscar Arias habría hecho esto en función de mantener su capital político internacional, dentro del cual el apoyo feminista no es escaso y se materializa a través de una entidad como la Fundación Arias-, incluso esto no es suficiente para explicar por qué Laura Chinchilla pudo ser candidata ni por qué ganó con el respaldo que obtuvo. 
chos humanos integrales de la población costarricense y particularmente, de las mujeres, en el marco de una democracia liberal. Ejemplos de esto han sido las decisiones de la Presidenta de no abrir a la discusión una reforma constitucional para separar a la Iglesia Católica del Estado, la constante exposición de sí misma como de confesión católica y su apoyo a los partidos cristianos minoritarios con asiento en la Asamblea Legislativa para impedir la igualdad de derechos civiles de las personas no heterosexuales.

Sin embargo, mientras que estas posturas son una reacción conservadora a los cambios que ocurren en la constitución de nuevas y diversas formas de familia, así como de vivir los géneros y las sexualidades, resulta difícil pensar que el pasado pueda, en algún momento, prevalecer y retomar el lugar del porvenir. La historia, si es que esta todavía existe, ha de tener memoria. Si ha saboreado los placeres de la libertad y de la dignidad, que da la autonomía, es poco probable que la memoria pueda extinguirse. Y esto es especialmente cierto para la historia y la memoria de las mujeres. Es posible que, si aceptamos las nuevas realidades y construimos nuevas formas de convivencia social, sin subordinaciones ni exclusiones y por el contrario, reconozcamos la diversidad humana, dejemos de vivir con esa sensación catastrofista, de fin de mundo, que está tan de moda. Porque lo que se está acabando, no necesariamente ha sido mejor, cuando proporcionó bienestar a costa de la libertad y del tiempo de las mujeres.

Un nuevo pacto sexual y social es necesario para que la convivencia pueda construirse en solidaridad y no en depredación. Esto implica que las mujeres ya no podrán ser pactadas, sino que su propia palabra y deseos, incluso cuando sean opuestos a las prescripciones sociales de la maternidad, tendrán que ser escuchadas y respetadas.

\section{BIBLIOGRAFÍA}

LIBROS

AGEM, UNIFEM, INEC, INAMU, PNUD. Las brechas de género en Costa Rica. Compendio de indicadores de género. 2010.
Arriagada, I. (Coord.). "Familias y políticas públicas en América Latina: una historia de desencuentros". Libros de la CEPAL 96. Octubre 2007.

Bourdieu, P. y L. J.D. Wacquant. Respuestas por una antropología reflexiva. México. Grijalbo, 1995.

Castoriadis, C. "La institución imaginaria de la sociedad". Volumen segundo. El imaginario social y la institución. Barcelona. Tusquets, 1989: 283-334.

Flórez-Estrada, M. De "ama de casa" a mulier economicus. Sexo, género, subjetividad y economía en Costa Rica contemporánea. San José, CR. Editorial de la Universidad de Costa Rica, 2010.

Flórez-Estrada, M. Economía del género. El valor simbólico y económico de las mujeres. San José, CR. Editorial de la Universidad de Costa Rica, 2010.

Foucault, M. Las palabras y las cosas. $16^{\mathrm{a}}$. Edición. Siglo Veintiuno Editores, 1985.

Foucault, M. El orden del discurso. Tusquets Editores. Octubre, 1999.

Marx, C. El Capital. Libro II, tomo II, Akal. 1997: 220.

Montiel M., N. "Crecimiento económico, productividad laboral y empleo asalariado: un análisis sectorial". Ulate Quirós, Anabelle, compiladora. En: Empleo, crecimiento y equidad: los retos de las reformas económicas de finales del siglo XX en Costa Rica. San José. CR. Editorial de la Universidad de Costa Rica. CEPAL, 2000: 263-292.

Lizano, E. Ajuste y crecimiento en la Economía de Costa Rica 1982-1994. San José, Costa Rica: Academia de Centroamérica. Estudios 13. 1999.

Raventós Vorst, C. Abstencionistas en Costa Rica: ¿Quiénes son y por qué no votan? San José, CR. Editorial de la Universidad de Costa Rica. IIDH/CAPEL, 2005.

Scott W., J. "La mujer trabajadora en el siglo XIX". Georges Duby y Michelle Perrot. Historia de las Mujeres. El siglo XIX, cuerpo trabajo y modernidad 4. Madrid. Taurus. Grupo Santillana de Editores SA., 2000:427-461. 
Vega, I. y A. Cordero. Realidad familiar en Costa Rica. San José, Costa Rica. UNICEF, FLACSO, IIP-UCR. 2001.

\section{REVISTAS}

Gamboa Barboza, I. "La pobreza como desolación: vivencias y representaciones en hombres y mujeres rurales". Anuario de Estudios Centroamericanos 35 (en prensa). Universidad de Costa Rica, 2009.

Polletta, F. y J.M., Jasper. "Collective Identity and Social Movements". JSTOR, Annu. Rev. Sociol. 27. New York. Department of Sociology, Columbia University. 2001: 283-305.

\section{TEXTOS ELECTRÓNICOS}

ANEP. 10 medidas para enfrentar la crisis económica con inclusión social y productiva. 2009. En: <http://www.anep. or.cr /leer.php/2914> [consultado el 15 de octubre de 2010].

Archivo Electoral. Plan de Gobierno, Laura Chinchilla. 2010. En: <http://www. archivoelectoral.org/documentos/11> [consultado el 14 de octubre].

Barciela, G. Leer Althusser o cuando las estructuras caminan por las calles. Instituto de Investigación Social, Económica y Política Ciudadana (ISEPCi) - Instituto de Altos Estudios Sociales, UNSAM. En: <http://www.isepci.org.ar> [consultado el 10/10/2010].

Bernanke, B. S. Semiannual Monetary Policy Report to the Congress. Before the Committee on Banking, Housing, and Urban Affairs, us Senate February 14, 2007. En: <http://www.federalreserve. gov $>$ [consultado el 5 de abril].

Chinchilla, L. La familia y el cuidado de dependientes. 2009. En: <http://www. nacion.com/ln_ee/2009/agosto/17/ opinion2060592.html> [consultado el 23/10/10].

CIEP-UCR. Encuesta telefónica. Elecciones 2010. Informe de Resultados. 2010. En: <http:www.ucr.ac.cr/ medios/documentos/Encuesta_
Postelectoral_2010.pdf.Febrero $>$ [consultada el 2 de octubre de 2010].

Espinoza, J. R. "Liberación Nacional y las mujeres". 2009. En: <http://www. nacion.com/ln_ee/2009/diciembre/17/ opinion2197140.html> [consultado el 23/10/10].

Informatico. Feministas no apoyan candidatura de Laura Chinchilla. 2010. En: <http://www.informa-tico.com/ index.php? scc $=$ articulo \& edicion $=2009$ $102 \&$ ref $=2610-090001>$ [consultado el 23/10/10].

Jameson, F. y S. Zizek. "Estudios culturales. Reflexiones sobre el multiculturalismo" Espacios del Saber 6. Cap. I. Argentina. Paidós, 1998: 69-136. En: <http://www. cholonautas.edu.pe> [consultado el 10/10/10].

Krugman, P. "Falling Wage Syndrome". New York Times. 2009. En: <http:// www.nytimes.com/2009/05/04/ opinion / $04 \mathrm{krugman}$.html? e m > [consultado el 4 de mayo].

InAmu. Plan de Acción 2008-2010 de la Política Nacional de Igualdad y Equidad de Género. 2008. En: <http://www. inamu.go.cr/content/view/812/1299/> [consultado el 30 de noviembre].

InEc. Encuesta de Hogares de Propósitos Múltiples. Principales Resultados. Varios Años. 2010. En: <http://www.inec.go.cr> [consultado el 7 de octubre].

Marx, C. El Capital. Tomo I. 2010. [Libro en línea] En: <http://www.ucm.es/info/bas/ es/marx-eng/capital11> [consultado el 21/10/10].

Mata, A. Gobierno dedicará \$32.000 millones para redes de cuido. 2010. En: <http:// www.nacion.com $>$ [consultado el 5/10/10].

OIT. Día Internacional de la Mujer 2010. Más mujeres eligen trabajar, pero la igualdad de género en el trabajo aún está muy lejos. 2010. En: <http://web.oit.or.cr/ index.php?option $=$ com_content\&task $=v$ iew\&id $=570 \&$ Itemid $=0>$ [consultado el 26/03/10]. 
OIT. Evolución de los salarios en América Latina 1995-2006. Santiago, octubre de 2008. En: <http://www.oit.org> [consultado el 7/10/10].

Rodríguez, I. Consumo de frijoles disminuye un $35 \%$ en últimos 45 años. En: <http://www.nacion.com/2010-09-17/ AldeaGlobal/NotaPrincipal/AldeaGlobal 2524055. aspx> [consultado el 17/09/10].

Tribunal Supremo de Elecciones (TSE). Elecciones en cifras 1953-2010. En: $<$ http://www.tse.go.cr/elecciones.htm> [consultado el 20/10/10].

Vizcaíno, I. Lo que viene es mi gobierno, no el de Óscar Arias. 2010. En: <http:// wvw.nacion.com/ln_ee/2010/enero/21/ pais2232180.html> [consultado el 21/01/10].

OTROS

ADC. "Estimación del aborto inducido en Costa Rica, 2007”. Informe de resultados. Junio, 2008.

Associated Press. EEUU: Reserva Federal mantiene tasas de interés. 28 de junio, 2007.

CCP e INEC. Costa Rica: estimaciones y proyecciones de población por sexo $y$ edad (cifras actualizadas) 1950-2010. San José, Costa Rica: publicaciones del Instituto Nacional de Estadística y Censos (INEC). 2008.

CEPAL. Transformaciones demográficas y su influencia en el desarrollo de América Latina y el Caribe 268. Naciones Unidas, 2008.

Flórez-Estrada, M. "Transformaciones en los roles femeninos y masculinos $y$ convivencia en Costa Rica”. Informe final de investigación para el Informe Nacional de Desarrollo Humano. Programa de las Naciones Unidas para el Desarrollo. 2009.

INEC. Encuesta Nacional de hogares, empleo y desempleo. Noviembre, 1979.

Laure, J. "Costa Rica: medio siglo de políticas a favor del incremento de salarios mínimos más bajos. Consecuencias sobre el poder de compra general y del alimentario" Colección Documentos Técnicos 19. Ciudad de Guatemala, Guatemala: Instituto de Nutrición de Centro América y Panamá (INCAP), 1990.

Román, I. y Morales, N. Demanda potencial de cuido infantil y estructura de los hogares: el caso de Costa Rica. InAmu, Programa Estado de la Nación-CONARE. 2010.

Fecha de ingreso: $21 / 01 / 2011$ Fecha de aprobación: 25/04/2011 
